\title{
A QoS-Based Multichannel MAC Protocol for Two-Tiered Wireless Multimedia Sensor Networks
}

\author{
GholamHossein EkbataniFard, Mohammad H. Yaghmaee, Reza Monsefi \\ Faculty of Engineering, Ferdowsi University of Mashhad, Mashhad, Iran \\ E-mail: \{Ekbatanifard,Yaghmaee\}@ieee.org,Monsefi@um.ac.ir \\ Received March 8, 2010; revised April 24, 2010; accepted May 27, 2010
}

\begin{abstract}
Rapid penetration of small customized wireless devices and enormous growth of wireless communication technologies have already set the stage for large-scale deployment of wireless sensor networks. Offering precise quality of service (QoS) for multimedia transmission over sensor networks has not received significant attention. However offering some better QoS for wireless multimedia over sensor networks raises significant challenges. In this paper, we propose an adaptive Cross-Layer multi-channel QoS-MAC protocol to support energy-efficient, high throughput, and reliable data transmission in Wireless Multimedia Sensor Network (WMSNs). Our proposed protocol uses benefit of TDMA and CSMA/CA to adaptively assign channels and timeslots to active multimedia sensor nodes in clusters. Simulations show that the proposed system achieves the performance objectives of WMSNs with increased network throughput at the cost of a small control and energy overhead.
\end{abstract}

Keywords: Wireless Multimedia Sensor Networks, MAC, Multichannel, Cross-Layer, Cluster, Adaptive

\section{Introduction}

The main component of wireless (multimedia) sensor network, are the sensor nodes, which are small in size, capable of self-organizing, sensing, processing data and communicating with other nodes. The availability of inexpensive hardware such as CMOS cameras and microphones that can ubiquitously capture multimedia content from the environment has fostered the development of Wireless Multimedia Sensor Networks [1], i.e., networks of wirelessly interconnected devices that can retrieve video and audio streams, images, and scalar sensor data.

The major objectives behind the research and deployment of sensor networks [2] lie in the following two broad aspects:

1) Event detection and possible data acquisition by sensing, data processing and communication through node coordination and data transmission $[3,4]$ to the sink or to the interested user.

2) Conservation of energy [5] to maximize the post deployment, active lifetime of individual sensor nodes and the overall network. The reason is that replenishing the energy of sensor nodes by battery-replacement is clearly not feasible for a large network consisting of hundreds of nodes. Moreover, wireless sensors are often deployed in an area which is in-approachable to humans and away from any sustained power-supply.

On the other hand, today's wireless communication is a gradually changing paradigm from its existing voice-alone services to a new world of real-time audio-visual applications.

This ever-increasing popularity of multimedia applications has already started penetrating the domain of wireless sensor networks-thereby giving birth to the new terminology wireless multimedia sensor networks [6].

Video surveillance, telemedicine and traffic-control are going to be the killer-applications of these emerging WMSNs. While the need to minimize the energy consumption has driven most of the existing research in wireless sensor networks, these new applications require the sensor network paradigm to be re-investigated in view of application-specific quality of service (QoS).

A quick look into the existing MAC protocols for sensor networks reveals that lack of standardization and application-specific diverse requirements has deprived wireless sensor networks from having a single de-facto standard MAC protocol. Most of the existing MAC protocols for wireless sensor networks can be divided into two categories: 1) time division multiple access (TDMA)-based and 2) carrier sense multiple access (CSMA) based with (possible) collision avoidance (CA) [7].

TDMA protocols have a natural advantage of colli- 
sion-free medium access; CSMA-CA protocols have a lower delay at varying traffic loads. However, transmitting multimedia applications with QoS offers significant new challenges over these energy-constrained sensor networks. Design of an efficient sensory MAC protocol, satisfying QoS requirements, is one major step in end to end QoS provisioning over WMSNs.

Current sensor nodes, such as MICAz and WINS, already support multiple channels for communication, for example, 40 channels in WINS [1].

Thus, by developing a multichannel MAC protocol, which can effectively utilize the available channel capacity through the cooperative work from other sensor nodes, we can achieve a better support for multimedia applications which demand for high data rates [8].

This motivates us to look for QoS-based, yet energy-aware, MAC protocols for WMSNs. The objective of this work is to develop a new QoS-based, energy-aware MAC protocol for WMSNs. In this paper, we propose an adaptive cross-layer multichannel protocol for MAC layer in WMSNs. This protocol use benefits of TDMA and CSMA/CA techniques in one MAC protocol.

The rest of the paper is organized as follows. Section II reviews existing works in sensory MAC protocols. Subsequently, in Section III we explain our proposed MAC protocol at some sub sections. Simulation results in Section IV corroborate the efficiency of the protocol in achieving the desired throughput and delay. Section $\mathrm{V}$ concludes the paper.

\section{Related Work}

A good survey of major MAC protocols for wireless sensor networks is provided in [9]. Provisioning QoS in MAC layers for wireless cellular and local area networks [10] is an active research area, QoS-based MAC protocol for wireless sensor networks have received relatively less attention. While both TMAC [11] and DSMAC [12] attempt to reduce the latency, little of the other MAC protocols are developed with an objective to optimize (or improve) some application-specific quality of service (like delay, throughput etc).

Protocols, like SPEED [13], cluster-QoS [14] and delay-constrained least cost routing [15] discuss the QoSrouting issues in wireless sensor networks. Unfortunately, all these works attempt to optimize QoS in the sensorrouting from higher layers only. However, end-to-end QoS in WMSNs cannot be satisfied without designing an efficient QoS-aware MAC protocol. Unfortunately only a handful of works exist for QoS-MAC in wireless sensor networks. These include Q-MAC [16], PQ-MAC [17], and RL-MAC [18]. To the best of our knowledge COM-MAC [8] is the first one that use multi channel techniques for MAC protocol in WMSNs. But it uses static time slots at control channel and doesn't propose any mechanism for nodes that do not have data for sending at start of intervals or for nodes that start sending data between an interval that these could increase delay and degrades throughput of the network.

This motivates us to develop a new cross-layer multichannel QoS-aware MAC protocol for clustered WMSNs that adaptively changes the intervals and use dynamic nature at channel and time slot assigning, therefore promoting the throughput of the network and exploit the high energy efficiency.

\section{Design of the Proposed Protocol}

\subsection{Network Architecture}

As shown in Figure 1, a WMSN consists of several more powerful nodes as cluster heads that located at the center of different monitoring area, a number of identical and stationary multimedia sensor nodes surrounding each cluster head and a remote data sink which stores the multimedia content locally for later retrieval. Each sensor node can communicate directly with its cluster head and cluster head can communication directly with the data sink using an out-of-band channel. But, if direct communication is not available, multi-hop routing is also employed.

\subsection{Our Assumptions}

We make some assumptions with relation to the configuration of the network. These assumptions are:

Topology of network is cluster based.

There are $\mathrm{N}$ different channels available for use and all channels have the same bandwidth except one that has lesser bandwidth than others and use as reserved channel, namely channel-R.

Cluster heads can transmit or receive on $\mathrm{N}$ channels at the same time.

Cluster heads will have sufficient power supply and more processing capacity than other sensor nodes.

All multimedia sensor nodes in a cluster can transmit or

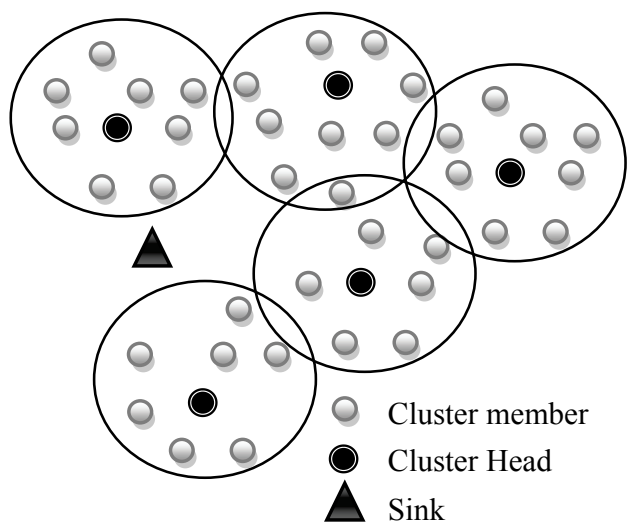

Figure 1. Network architecture. 
receive on three channels, namely channel-1 and channel-2 and channel-R. Channel- 1 is a contention based channel that assigned by cluster head at first phase of network deployment. Channel-2 is contention free and dynamically assigned by cluster head. Channel- $\mathrm{R}$ is a contention based reserved channel that is share between a cluster members. Sensor nodes are able to switch among channels dynamically. The channel switching time is less than $224 \mu$ s according to [1].

The working of a cluster of sensor nodes is synchronized to the cluster head and each sensor node can communicate directly with its cluster head.

\subsection{Proposed QoS-MAC Protocol}

We assume that the clustering process has been completed by performing a clustering protocol, the assignment of sensor nodes to clusters can be handled by existing clustering techniques [2]. Within each cluster, all tasks are done in time intervals $(\Delta \mathrm{T})$, which are dynamically changed. $\Delta \mathrm{T}$ as Time interval can be varying depending on application and traffic load of the network.

We suppose three type nodes in a cluster as illustrated in Figure 2. These nodes are: cluster head, active nodes which are nodes that have data for sending, and passive nodes which have not data for sending at present. First of all, when the network is initially deployed, channel-1 allocation phase begin in each cluster and doing only one time. Channel-1 will be used as control channel for sending request message from multimedia sensor nodes to cluster head. As mentioned before the number of channels at cluster head is limited. So, it may happen that a channel assigned to more than one sensor nodes in a cluster. N-1 channels of cluster head could be assigned at this phase as channel-1 of multimedia sensor nodes. One remained channel from $\mathrm{N}$ channels of cluster head will be used as channel-R. That is share between all of nodes in a cluster. The usage of channel-R will be expressed later.

The operations of a cluster on $\Delta \mathrm{T}$ are organized in three sequential phases: request phase, scheduling phase and data transmission phase. We now explain our MAC protocol details in three phases.

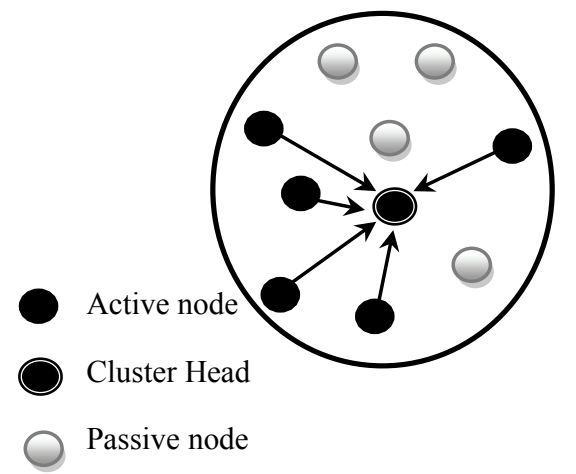

Figure 2. Node types in a cluster.

\subsubsection{Request Phase}

After channel-1 assignment phase, that runs only one time, the network operations begin at $\Delta \mathrm{T}$ intervals. At the start of each $\Delta \mathrm{T}$, the network layer of each multimedia sensor node determines that whether information exists for sending or not in a cross layer manner. Then nodes that have information for sending, active nodes, start request phase on channel-1, and send a request message (REQ) to the cluster head.

The REQ message includes QoS requirements, such as amount of multimedia data to be transmitted, maximum delay, priority information and traffic class (streaming video, Non-Real Time (NRT), Best Effort (BE)), and Packet Error Rate (PER).

Because channel-1 may be assigned to more than one node in a cluster, so, adaptive contention window protocol [19] can be used for better performance on this channel.

When active nodes received acknowledgement of its REQ message, they go to standby mode and waiting for scheduling message from cluster head.

Request phase time $(\Delta \mathrm{Tr})$ determined dynamically, based on event rate, traffic load and average of previous $\Delta \operatorname{Tr}$ periods.

\subsubsection{Scheduling Phase}

After request phase, cluster heads gather REQ messages and then start scheduling phase. Each cluster head calculate an appropriate schedule, based on priority and other QoS requirements that specified in REQ messages, to coordinate the data transmissions of active nodes. Then cluster heads broadcast scheduling messages through all N-1 channels.

In scheduling message, cluster head assign a channel as channel-2 to each active node for data transmission. If the number of active nodes in a cluster is more than $\mathrm{N}-1$, then a channel should assign to more than one active node. In fact, a time slot in a channel may be assign to an active node as channel- 2 .

Therefore, the scheduling message includes a channel and probably a time slot in it as channel-2 for each active node. Moreover new $\Delta \mathrm{T}$ that calculated based on REQs is included in scheduling message. New $\Delta \mathrm{T}$ specifies end of this interval and start of next interval indeed. A cluster time intervals have been illustrated in Figure 3 for three intervals.

Lengths of time slots that assign to active nodes are depending on amount of data that specified in REQ me-

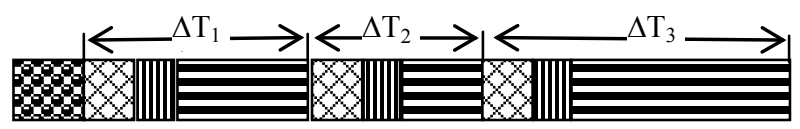

Channel-1 assignment phase

Request phase $(\Delta \mathrm{Tr})$

Scheduling phase (chanel-2 assignment phase)

Data transmission phase

Figure 3. A cluster operations for three intervals. 
ssages and times need for sending acknowledgment messages if needed. Some traffic types may not require acknowledgement message from cluster head, that it should be declared in REQ message.

The priority of REQs is essentially based on its traffic class, low priority for best effort traffic, medium priority for non-realtime and high priority for streaming video traffics.

Figure 4 shows the pseudo-code of scheduling algorithm that assigns channel-2 and timeslots to active nodes in a cluster. Also announce new_ $\Delta \mathrm{T}$ for next interval. Thereafter, scheduling message broadcasts for sensor nodes in the cluster at network.

If some nodes that were passive get active after this phase, send their REQ messages to cluster head on channel-R. Then, if there is enough unused time on channels, it could be assigned to these nodes. Otherwise, if there is not enough time for some of these nodes, then these nodes only will be announced with new $\Delta \mathrm{T}$. So unsuccessful nodes will go to sleep mode until starting of next request phase.

The network throughput in an interval is given by

$$
\tau_{j}=\sum_{i=1}^{k} P_{i} /\left((N-1) T_{j} C\right)
$$

where $k$ is the total number of REQ messages in $j$ th interval, $P_{i}$ is amount of bits of data requested for transmission in $i$ th REQ message. Maximum occupied channel time in $j$ th interval is $T_{j}$ seconds. In other word $T_{j}$ is equal to new_ $\Delta \mathrm{T}$ at $j$ th interval that used in Figure 4.

$\mathrm{N}-1$ is the number of contention free channels available at cluster head. And capacity of each these channels are $C$ bps. The total throughput of network in $m$ time intervals is the average of $\tau_{j}$ for $j=1,2 \ldots m$.

\subsubsection{Transmission Phase}

After receiving a scheduling message by an active node, it could send its data on assigned channel. If a time slot in a channel is assigned to an active node, it could go to sleep mode until its time slot for sending data approaches.

As mentioned before, some passive nodes may get active at this phase, and send its REQ messages at channel-R. So, if free channels or time slots in channels has been assigned to such nodes, they could send its data at scheduled time.

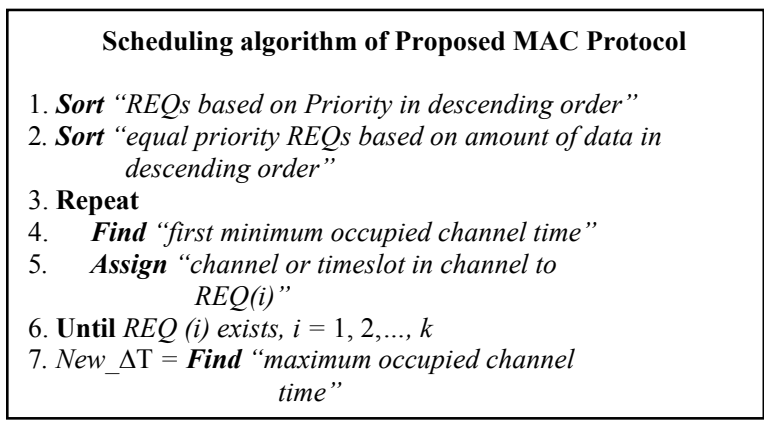

Figure 4. Scheduling algorithm.
When cluster head receives packets from its cluster members, it classifies traffics based on its priority then schedules it for sending toward the sink. Such framework illustrated in Figure 5.

Nodes receive acknowledgement messages (ACK) for proper sent packets, if specified before at REQ message. Nodes could request unused timeslots, if exist, in an interval for lost packets.

As mentioned earlier the streaming video traffic is assigned the highest priority and the best effort traffic is assigned the lowest priority. We will now analyze the average delay incurred in each of this traffic class. The mean waiting time of a type $i$ customer is denoted by $E\left(W_{i}\right)$ and $E\left(L_{i}^{q}\right)$ is the number of type $i$ customers waiting in the queue. Further let's assume the processing time of traffic class $i$ is $\mu_{i}$, with mean $E\left(\mu_{i}\right)$ and residual processing time $\left(R_{i}\right)$, with mean $E\left(R_{i}\right)$. Then the traffic intensity of the system is given by: $\rho_{i}=\lambda_{i} E\left(u_{i}\right)$ [12]. Hence, for the highest priority streaming video traffic it holds that

$$
\begin{aligned}
& E\left(W_{1}\right)=E\left(L_{1}^{q}\right) E\left(\mu_{1}\right)+\sum_{j=1}^{r} \rho_{j} E\left(R_{j}\right) \\
& =\frac{\sum_{j=1}^{r} \rho_{j} E\left(R_{j}\right)}{1-\rho_{1}}
\end{aligned}
$$

where $r$ is the number of different traffic classes whose service is in progress during the arrival of the highest priority traffic class.

And the mean waiting time for lower priority traffics [7] could be estimated as

$$
\begin{gathered}
E\left(W_{i}\right)=\frac{\sum_{j=1}^{i} E\left(L_{j}^{q}\right) E\left(\mu_{j}\right)+\sum_{j=1}^{r} \rho_{j} E\left(R_{j}\right)}{1-\left(\rho_{1}+\cdots+\rho_{i-1}\right)} \\
\forall i: 2 \leq i \leq n .
\end{gathered}
$$

\section{Performance Evaluation}

In this section we show different simulation results demonstrating the efficiency of this proposed MAC protocol. We have developed a discrete-event object oriented packet-level simulator in $\mathrm{C}++$. In the simulations presented in this section, the considered packet size is 25 bytes. We assume 3 data channels at sink that each channel capacity is $250 \mathrm{kbps}$. Experiments are repeated 10 times. The performance of our algorithm is compared with COM-MAC

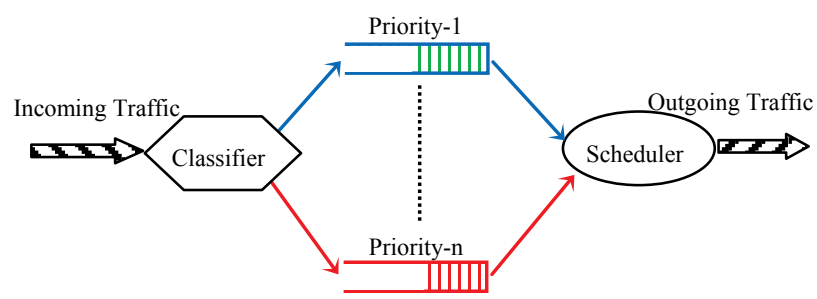

Figure 5. Traffic differentiation and priority queuing in WMSN. 
[8] and a baseline protocol, the multichannel TDMA (M-TDMA) protocol. For M-TDMA, the cluster head first evenly distribute the cluster members on the available channels. Then, the cluster head generates a TDMA schedule on each channel and allocates a fixed slot to each cluster members.

Figure 6 compares the network throughput performance of proposed MAC protocol with COM-MAC and MTDMA for different cluster sizes. Our protocol works well. But, when the number of nodes in cluster increases, the proposed protocol throughput decreases. To find the reason we repeat simulation up to 100 nodes in a cluster, Figure 7, I see that our protocol throughput reach to a steady state and it has better throughput than other protocols. This is because the channel tends to be saturated when more nodes are trying to utilize the channel.

As expected, proposed MAC protocol outperforms other two protocols. This is because our protocol is designed to maximize the network throughput by adaptively changing intervals and using unused channels and time slots for passive nodes that get active after request phase.

Figure 8 shows the delay performance compassion of three protocols as cluster size increases. We see that our proposed MAC incurs lower delay when compared to COM-MAC and M-TDMA.

It is because that in our protocol, when a passive node gets active, it could send request from channel-R to use unassigned space on channels for data transmission. So it incurs lower delay than other two protocols. With COMMAC and M-TDMA such nodes should wait until next intervals thus this increases packet delay. We also notice that the delay performance increases as cluster size increases. This is because that larger cluster size will lead to heavier network load so that a packet has to wait longer to be transmitted.

Figure 9 explains the throughput-dynamics for different traffic classes. The novelty of our MAC protocol is that it first classifies the traffic into different classes depending on the type of service (ToS) then schedules it for data transmission. The streaming video traffic is given the highest priority, the NRT traffic is given the second priority and

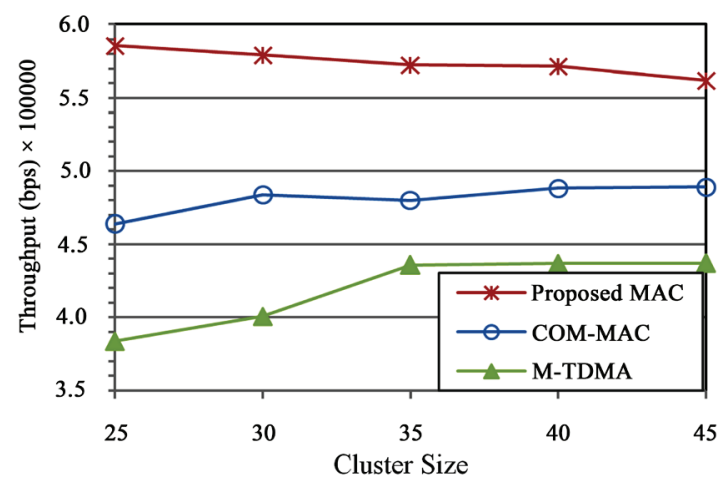

Figure 6. Throughput performance for various cluster sizes up to 45 .

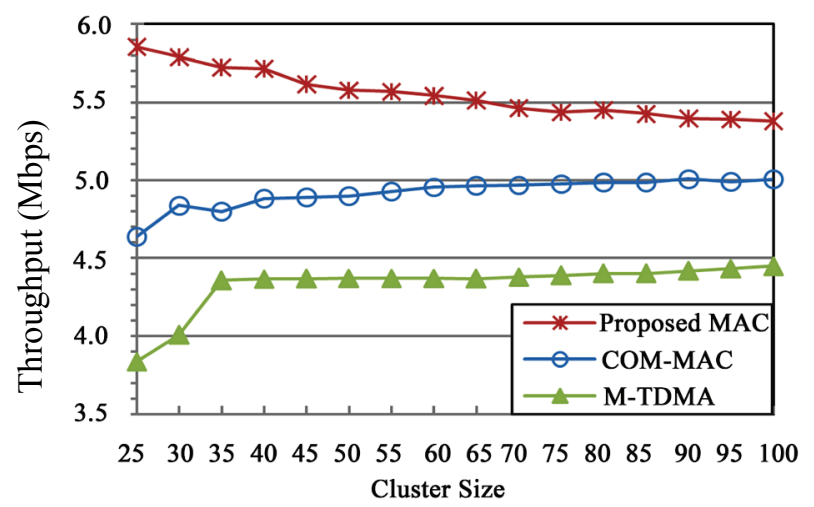

Figure 7. Throughput performance for various cluster sizes up to 100 .

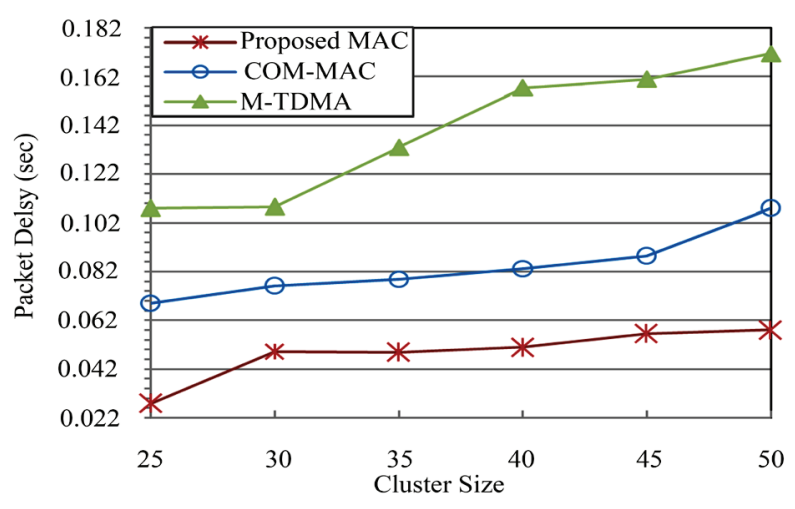

Figure 8. Packet delay performance for various cluster.

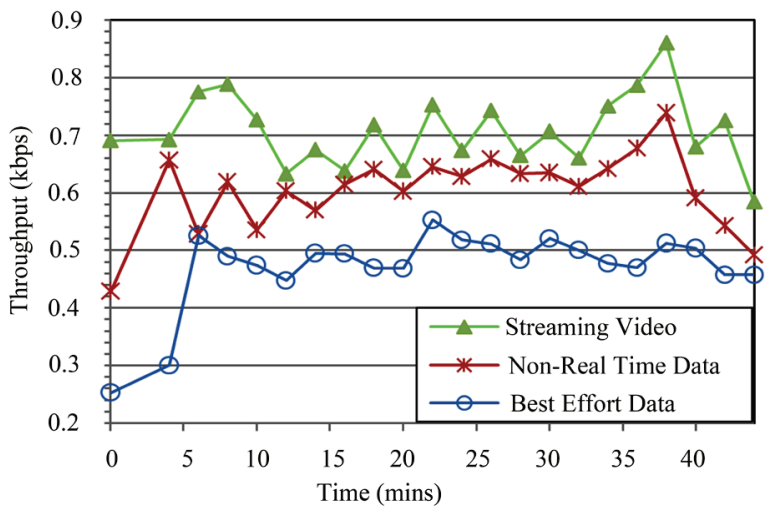

Figure 9. Differentiated MAC-throughput.

the BE traffic attains a lowest throughput.

\section{Conclusions}

In this paper we have developed a cross layer multichannel QoS-MAC protocol for wireless multimedia sensor networks which classifies the wireless traffic into different class, and adaptively assigns channel to various traffics. In our proposed protocol nodes get active only when the network layer specifies that there are data for sending. We 
verify the advantages of our protocol through network simulation, in terms of network delays, throughput and differentiated throughput of different traffic classes. We see that our proposed MAC protocol provides better energy-efficiency, high-throughput, and data reliability support in WMSNs.

\section{Acknowledgements}

This work was supported in part by grants from Faculty of Engineering Ferdowsi University of Mashhad under the contracts 18069 .

\section{References}

[1] I. F. Akyildiz, T. Melodia and K. R. Chowdury, "A Survey on Wireless Multimedia Sensor Networks," Computer Networks (Elsevier), Vol. 51, No. 4, 2007, pp. 921-960.

[2] I. F. Akyildiz, W. Su, Y. Sankarasubramaniam and E. Cayirci, "A Survey on Sensor Networks," IEEE Communications Magazine, Vol. 40, No. 8, 2002, pp. 102-114.

[3] W. Heinzelman, J. Kulik and H. Balakrishnan, "Adaptive Protocols for Information Dissemination in Wireless Sensor Networks," Proceedings of the 5th Annual ACM/ IEEE International Conference on Mobile Computing and Networking, Seattle, WA, August 1999, pp. 174-185.

[4] Y. Yao and J. Gehrke, "The COUGAR Approach to In-Network Query Processing in Sensor Networks," $A C M$ SIGMOD Record, Vol. 31, No. 3, 2002, pp. 9-18.

[5] R. A. F. Mini, M. do V. Machado, A. A. F. Loureiro and B. Nath, "Prediction-Based Energy Map for Wireless Sensor Networks," Ad Hoc Networks, Vol. 3, No. 2, 2005, pp. 235-253.

[6] I. F. Akyildiz, T. Melodia and K. R. Chowdhury, "A Survey on Wireless Multimedia Sensor Networks," The International Journal of Computer and Telecommunications Networking, Vol. 51, No. 4, 2007, pp. 921-960.

[7] N. Saxena, A. Roy and J. Shin, "Dynamic Duty Cycle and Adaptive Contention Window Based QoS-MAC Protocol for Wireless Multimedia Sensor Networks," Computer Networks (Elsevier), Vol. 52, No. 13, 2008, pp. 25322542.

[8] C. Li, P. Wang, H.-H. Chen and M. Guizani, "A Cluster Based On-demand Multichannel MAC Protocol for Wireless Multimedia Sensor Networks," IEEE International Conference on Communications, Beijing, May 19-23, 2008, pp. 2371-2376.
[9] I. Demirkol, C. Ersoy and F. Alagoz, "MAC Protocols for Wireless Sensor Networks: A Survey," IEEE Communications Magazine, Vol. 44, No. 4, 2006, pp. 115-121.

[10] T. Kuhn, "A QoS MAC Layer for Ambient Intelligence Systems," Proceedings of the 4th International Conference on Pervasive Computing, Dublin, 2006, pp. 69-72.

[11] T. V. Dam and K. Langendoen, "An Adaptive Energy-Efficient MAC Protocol for Wireless Sensor Networks," Proceedings of the 1st International Conference on Embedded Networked Sensor Systems, Los Angeles, 2003, pp. 171-180.

[12] L. Kleinrock, "Queueing Systems, Theory,” John Wiley \& Sons, New York, 1975.

[13] T. Hea, J. A. Stankovica, C. Lub and T. Abdelzahera, "SPEED: A Stateless Protocol for Real-Time Communication in Sensor Networks," 23rd IEEE International Conference on Distributed Computing Systems, Rhode Island, USA, May 2003, pp. 1-10.

[14] S. S. Tang and W. Li, "QoS Supporting and Optimal Energy Allocation for a Cluster Based Wireless Sensor Network," Computer Communications, Vol. 29, No. 13-14, 2006, pp. 2569-2577.

[15] Q. Gao, K. J. Blow, D. J. Holding, I. Marshall and X. H. Peng, "Radio Range Adjustment for Energy Efficient Wireless Sensor Networks," Ad-Hoc Networks, Vol. 4, No. 1, 2006, pp. 75-82.

[16] Y. Liu, I. Elhanany and H. Qi, "An Energy-Efficient QoS-Aware Media Access Control Protocol for Wireless Sensor Networks," IEEE International Conference on Mobile Adhoc and Sensor Systems, Washington, DC, November 7, 2005, pp. 191-193.

[17] K. Paek, J. Kim, U. Song and C. Hwang, "Priority-Based Medium Access Control Protocol for Providing QoS in Wireless Sensor Networks," IEICE Transaction Letters on Information Systems, Vol. E90-D, No. 9, 2007, pp. 14481451.

[18] Z. Liu and I. Elhanany, "RL-MAC: A QoS-Aware Reinforcement Learning Based Mac Protocol for Wireless Sensor Networks," IEEE International Conference on Networking, Sensing and Control, Lauderdale, 2006, pp. $1-6$.

[19] N. Sabena, A. Roy and J. Shin, "Dynamic Duty Cycle and Adaptive Contention Window Based QoS-MAC Protocol for Wireless Multimedia Sensor Networks," Computer Networks, Vol. 52, No. 13, 2008, pp. 2532-2542. 